\title{
Laparoscopic Cholecystectomy Complication and Conversion Rate
}

\author{
Malla $\mathrm{BR}^{1}$, Shrestha RKM ${ }^{2}$ \\ ${ }^{1-2}$ Department of Surgery, \\ Dhulikhel Hospital - Kathmandu University \\ Hospital, \\ Dhulikhel, Nepal
}

\section{Corresponding Author}

Dr Bala Ram Malla

Department of Surgery

Dhulikhel Hospital - Kathmandu University Hospital

Dhulikhel, Nepal

Email.mallabr504@yahoo.com

Kathmandu Univ Med J 2010;9(32):367-9

\begin{abstract}
Background

Laparoscopic cholecystectomy has become standard method for treating gallstone. However, different centres have reported different complications and conversion rate. The objective of this study was to evaluate complications and conversion of laparoscopic cholecystectomy into open cholecystectomy in Dhulikhel Hospital, Kathmandu University, Nepal.
\end{abstract}

\section{Methods}

Files of all patients who had laparoscopic cholecystectomy from January 2005 to December 2009 were reviewed. Out of 119 laparoscopic cholecystectomy cases, 102 were included in the study as complete information was lacking in the rest.

\section{Results}

Out of 102 cases, 80 were female. Symptomatic cholelithiasis were $76.47 \%$. The mean hospital stay was 2.48 days. Postoperative complications occured in $5.88 \%$ patients. Conversion rate to open cholecystectomy was $3.92 \%$.

\section{Conclusions}

Laparoscopic cholecystectomy is a reliable and safe surgery. With growing experience in laparoscopic technique, it is possible to bring complications and conversion rate to minimum. However, there will be no significant improvements once learning curve is reached. Rather, the nature of biliary injury may become more severe.

\section{Key Words}

cholecystectomy, conversion, gallstone, laparascopic

\section{INTRODUCTION}

At present, laparoscopic cholecystectomy (LC) is the procedure of choice in the surgical treatment of the symptomatic biliary lithiasis. Laparoscopic cholecystectomy has become the standard operative procedureforthetreatmentofgallbladderdiseasesand almost replaced open cholecystectomy (OC) in the treatmentofgallbladderdiseases. ${ }^{1,2}$ Theoutcome of $L C$ is influenced greatly by training, experience, skill and judgmentofthesurgeonperformingtheprocedure. ${ }^{3}$ This studyanalyzed thecomplicationsandconversionrateof LC.

\section{METHODS}

Files of the patients, who had LC done from January 2005 to December 2009, were reviewed. Research was approvedbyinstitutionalreviewcommittee.Wecollected patients demographic data, indications of the surgery, related medical problems, history of previous surgery, preoperativeliverfunctiontest,reasonsfortheconversion and the postoperative complications. All the patients presentedwithcholelithiasiswithoutcholedocholithiasis; and withnocontraindicationforgeneralanaesthesiawere included in thestudy.Out of 119attempted cases ofLC, 102 cases were included in the study as the rest of the 
recordsweremissing.LCwasperformedusingtheclosed techniquewithstandardfourtrochars.Outof102cases of LC,fourcaseswereconverted toOC.Datawereanalyzed with Microsoft Excel.

\section{RESULTS}

Out of 102 cases, 80 (78.43\%) were female and 22 (21.56\%)male.Themeanagewas 42 years (range 16-72 years). One $(0.98 \%)$ patient had Hypertension and one had Diabetes Mellitus. The indications of laparoscopic cholecystectomyaregiveninTable1.Themeanhospital stay was 2.48 days (range 2-14 days).

Six patients (5.88\%) had postoperative complications. (Table 2)

AllthecasesofLCweregivensingleprophylacticdoseof cefotaxime1gramintravenously.InCasesofacutecalculous cholecystitis,empyemagallbladderandbilespillage,total threedosesofcefotaxime1gramweregivenintravenously. Fourpatients(3.92\%)outof102wereconverted toopen cholecystectomy. Reasons for conversion were frozen Calot's triangleintwopatients (1.96\%), bleedinginone (0.98\%)andconfusinganatomyatCalot'striangleinone (0.98\%) .

Table 1. Indications of laparoscopic cholecystectomy $(n=102)$

$\begin{array}{lc}\text { Diagnosis } & \text { No of cases (\%) } \\ \text { Symptomatic cholelithiasis } & 78(76.47 \%) \\ \text { Acute calculous cholecystitis } & 8(7.84 \%) \\ \text { Chronic calculous cholecystitis } & 14(13.74 \%) \\ \text { Empyema gallbladder with gall stone } & 2(1.96 \%) \\ \text { Total } & 102(100 \%)\end{array}$

Table 2. Postoperative complications in 102 laparoscopic cholecystectomy cases

\begin{tabular}{lc} 
Complications & No of cases (\%) \\
\hline Bile leak & $3(2.94 \%)$ \\
Wound infection & $1(0.98 \%)$ \\
Surgical emphysema & $1(0.98 \%)$ \\
& $1(0.98 \%)$ \\
\hline Bile duct injury & $\mathbf{6 ( 5 . 8 8 \% )}$
\end{tabular}

\section{DISCUSSION}

Laparoscopiccholecystectomyisnoteasyforthesurgeon. Laparoscopicsurgeryhaslearningcurve.Itneedthorough instructionaswellasexperiencefortheimprovementof result.

StudydonebyKeusetal ${ }^{4}$ showedmorbidityrates of $5.4 \%$ following LC. This rate is comparable to our morbidity rate of post LC (5.88\%). Eelco J Veen et al ${ }^{5}$ had also shown $7 \%$ post LC morbidity rates. However, the study has not included biliary injury rate (1\%). During the surgical learning curvefor LC, therewas an initial rise in thereportsofbileductinjuries, ${ }^{6}$ resultingmainlyfromthe surgeonsinexperienceandmisinterpretationofanatomy. However,LChasbeenstillassociated withsignificantbile ductinjuriesupto $0.5-0.8 \%{ }^{7-9}$ and thenatureofbileduct injury is more severe. ${ }^{10}$ An audit of $1522 \mathrm{LCs}$ performed in Thailand revealed a bile duct injury rate of $0.59 \% .{ }^{11}$ WaheebR.Al-Kubatietal ${ }^{12}$ showed biliaryinjuryin $0.6 \%$ of LC cases done for chronic calculous cholecysytitis.

In our study, bile duct injury was found in one patient $(0.98 \%)$ out of 102 cases. In this case, common hepatic ductwasclippedinsteadofcysticduct.Itwasdiagnosed on $7^{\text {th }}$ postoperatviveday and managed with Roux-n-y hepatico-jejunostomy.

The biliary leak may be minor, ${ }^{13}$ arising from a small, accessory bile duct ${ }^{14}$ and clinically insignificant. Percutaneousdrainageofthebilecollectedinsubhepatic spaceisusuallysufficientforsuchcases. Inourstudythere were3(2.94\%)caseswithbileleak.Subhepaticdrainkept duringoperationwassufficienttomanagebiliaryleakage. Inallcases, therewasminimal $(<50 \mathrm{ml})$ bile indrain. The drainwasremovedon4-5 ${ }^{\text {th }}$ postoperativedayinallcases.In caseswheretherewasdoubtaboutthehemostasisfromraw areaofgallbladderfossa,subhepaticdrainwaskeptduring operation.SimilarlyinthestudydonebyMuneerImran etal ${ }^{15}$, twopatients(8\%)hadbilestaineddrainfollowing laparoscopiccholecystectomyfor2days.Minorleakwas there in both cases and it was stopped spontaneously without requirement of any surgical intervention.

LC has become the first line of surgical treatment of calculousgallbladderdisease; however,conversiontoOC remainsa possibility. ${ }^{16}$ Ourconversion rateto OC in 102 cases of $L C$ was $3.92 \%$.

Table 3 comparesourconversion ratewith somemajor published similar work.

Table 3. Conversion rate of laprascopic cholecystectomy into open cholecystectomy

\begin{tabular}{lcc|}
\hline Study & No of patients & Conversion rate (\%) \\
\hline Saeed Hadi et al ${ }^{17}$ & 709 & 8.3 \\
\hline Waseen Memon et al ${ }^{18}$ & 216 & 4 \\
Butt et al $^{19}$ & 300 & 4 \\
Present study & 102 & 3.92
\end{tabular}

Saeed Hadiet al and Waseen Memon et al claimed that commonestcauseofconversionwasfrozenCalot'striangle which wastruein our studyalso. Frozen Calot'striangle 
means dense adhesion around Calot's Triangle

\section{CONCLUSION}

Laparoscopiccholecystectomyisareliableandsafesurgery. With growing experience in laparoscopic technique, it is possible to bring complications and conversion rate to minimum. However, there will be no significant improvementsoncelearningcurveisreached.Rather,the nature of biliary injury may become more severe.

\section{REFERENCES}

1. Nuri Aydin Kama, M. Kologlu, E. Reis, M. Atli, M. Dolapci. Risks score for conversion from laparoscopic to opencholecystectomy.TheAmerican JournalofSurgery 2001;181:520-5.

2. SinghK,OhriA.Laparoscopiccholecystectomy-Istherea need to convert? J Min Access Surg 2005;1:59-62.

3. LoCM, Fan ST, Liu CLetal.Early decision for conversion of laparoscopictoopencholecystectomyfortreatmentofacute cholecystitis. Am Jr Surg 1997;173:513-17.

4. KeusF,deJongJAF,GooszenHGetal.Laparoscopicversus open cholecystectomy for patients with symptomatic cholecystolithiasis (Review). [Internet] 2006 [cited 2010 Dec 10\}. Available from: http://www2.cochrane.org/ reviews/en/ab006231.html

5. VeenEJ,BikM,Janssen-HeijnenML,DeJonghM,Roukema AJ.Outcomemeasurementinlaparoscopiccholecystectomy byusing aprospectivecomplication registry:resultsofan audit. Int J Qual Health Care 2008;20:144-51.

6. HuangZQ,HuangXQ.Changing patternsoftraumaticbile duct injuries: A review of forty years experience. World J Gastroenterol. 2002;8:5-12.

7. Flum DR, Cheadle A, Prela C et al. Bile duct injury during cholecystectomyand survivalinmedicarebeneficiaries. JAMA 2003;290:2168-2173.

8. Nuzzo G, Giuliante F, Giovannini I et al. Bile duct injury duringlaparoscopiccholecystectomy:resultsofanltalian national survey on 56591 cholecystectomies. Arch Surg 2005;140:986-92.

9. Karvonen J, Gullichsen R, Laine S et al. Bile duct injury during laparoscopic cholecystectomy: primary and long-term results from a single institution. Surg Endosc 2007;21:1069-73.

10. Abou El-Ella KM, Mohamed ON, El-Sebayel MI, AlSemayerSA,AIMoflehIA.Managementofpostlaparoscopic cholecystectomy major bile duct injury: Comparison of MRCP withconventionalmethods.SaudiJGastroenterol 2004;10:8-15.

11. Sicklick JK, Camp MS, Lillemoe KD, Melton GB, Yeo CJ,Campbell KA, etal. Surgical management of bile duct injuriessustainedduringlaparoscopiccholecystectomy.Ann Surg. 2005;241:786-95.
12. Waheeb R. Al-Kubati: Bile Duct Injuries Following Laparoscopic Cholecystectomy:AClinical Study.Saudi J Gastroenterol. 2010;16:100-4.

13. MorgensternL,BerciG,PasternakEH.Bileleakafterbiliary tract surgery: A laparoscopic perspective. Surg Endosc. 1993;7:432-8.

14. Balija M, Huis M, Szerda F, Bubnjar J, Stulhofer M. Laparoscopiccholecystectomy-accessorybileducts.Acta Med Croatica. 2003;57:105-9.

15. Muneerl,ZahidM,Shahidl,KirinS,ShahTA.AComparative StudyofLaparoscopicVsopenCholecystectomyatGhurki TrustTeaching Hospital,Lahore.PakistanJournalOfMedical and Health Science 2010;4:419-22.

16. Slater K, Strong R, Wall D, Lynch S. latrogenic bile duct injury:theScourgeoflaparoscopiccholecystectomy.ANZJ-Surg 2002;72:83-8.

17. Al-Bahlooli SH, Al-Malahi A, Ghalla NH, Al-Dain AS, Ali Sabahi AA. Conversion rate of laparoscopic to open cholecystectomy. Yemeni Journal for Medical Science 2009;1:1-8

18. Memon W, Khanzada TW, Samad A, Laghari MH. Laparoscopic cholecystectomy: conversion rate and its causesat Isra UniversityHospital,Hyderabad.RMJ.2008; 33(2): 159-161.

19. Butt AU, Sadiq I: Conversion of laparoscopic to open cholecystectomy-sixyearsexperienceatShalamarHospital, Lahore. Ann King Edward Med Coll 2006;12:536-8. 